\title{
Strong Coupling in the Quantum Field Theory with Nonlocal Nonpolynomial Interaction
}

\author{
G. V. Efimov \\ Joint Institute for Nuclear Research, Laboratory of Theoretical Physics, Dubna, USSR
}

\begin{abstract}
In the relativistic quantum field theory the representation for the $S$ matrix elements is obtained for any coupling constants $g$ in the case of a one component scalar field $\varphi(x)$ with nonlocal nonpolynomial interaction $\mathscr{L}_{I}(\varphi)=g U(\varphi)$ when the causal function is bounded in the Euclidean region $0 \leqq D_{c}\left(x_{E}^{2}\right) \leqq D_{c}(0)<\infty$ and the function $|U(u)| \leqq 1$ for real $u$. It is proved that the two point Green function is bounded in the physical region of momenta variable $p^{2}$.
\end{abstract}

\section{Introduction. Formulation of the Problem}

Summation of the perturbation series in quantum field theory is now an outstanding problem in elementary particle physics. Summation of different classes of the Feynman diagrams does not solve the problem. Really we do not know the behaviour of total amplitudes for large coupling constants or high energies beyond the perturbation theory for any interaction lagrangians.

In the given paper we will obtain the representation for any matrix elements of the $S$-matrix for arbitrary coupling constants in the quantum field theory with nonlocal interaction for nonpolynomial interaction lagrangians. Moreover we will prove that the two point Green function is bounded for high energies. This conclusion is valid for other amplitudes of physical processes.

Earlier (see [1]) it was proved that in this quantum field model the $S$-matrix is covariant, finite, unitary and causal in each perturbation order. In this paper we will prove that for this model the total $S$-matrix satisfies the same conditions beyond the perturbation theory and its matrix elements are bounded for high energies.

We will consider the theory of a one component scalar field $\varphi(x)$, described by the following Lagrangian:

$$
\mathscr{L}(x)=\frac{1}{2} \varphi(x)\left(\square-m^{2}\right) \varphi(x)+g U\left(K\left(l^{2} \square\right) \varphi(x)\right) .
$$


Here $g$ is a coupling constant. The function $U(u)$ describing the interaction is real and analytic in a vicinity of the real axis, and such that

$$
\begin{aligned}
& \max _{-\infty<u<\infty}|U(u)|=1 \\
& U(u)=\int_{-\infty}^{\infty} d \beta \tilde{U}(\beta) e^{i \beta u} ; \int_{-\infty}^{\infty} d \beta|\tilde{U}(\beta)| e^{d|\beta|}<\infty \quad(\exists d>0) .
\end{aligned}
$$

Below we will consider two types of functions

(a) $U(u)=-U(-u) \quad$-odd functions

(b) $U(u)=U(-u) \quad$-even functions

We assume that $U(u) \geqq 0$ for $u \geqq 0$. The examples of such functions are

$$
u^{3} e^{-u^{2}}, \frac{u^{3}}{\left(1+u^{2}\right)^{2}}, u^{4} e^{-u^{2}}, \frac{u^{4}}{\left(1+u^{2}\right)^{3}}
$$

and so on.

The operator $K\left(\square l^{2}\right)$ in (1.1) is nonlocal. It means that the function $K(z)$ is an entire analytical function of a finite order $\varrho \geqq \frac{1}{2}$ in the complex $z$-plane and decreases rapidly enough when $z=p^{2} \rightarrow-\infty$ (in the Euclidean direction). The constant $l$ has the dimension of length and defines the scale of the nonlocal interaction.

All questions connected with the quantization of this system (1.1) and the construction of the $S$-matrix, which is covariant, finite, unitary and causal in each perturbation order, are expounded in [1].

The $S$-matrix as a functional of the scalar field can be written in the Euclidean metrics in the following way

$$
S[\varphi]=\exp \left\{\frac{1}{2} \iint d x_{1} d x_{2} D\left(x_{1}-x_{2}\right) \frac{\delta^{2}}{\delta \varphi\left(x_{1}\right) \delta \varphi\left(x_{2}\right)}\right\} \cdot \exp \left\{g \int d x U(\varphi(x))\right\}
$$

This representation should be understood as an expansion in the coupling constant $g$. Here $D\left(x_{1}-x_{2}\right)$ is the nonlocal causal function in the Euclidean metrics

$$
\begin{aligned}
& D\left(x_{1}-x_{2}\right)=K\left(\square_{1} l^{2}\right) K\left(\square_{2} l^{2}\right) \overline{\varphi\left(x_{1}\right) \varphi}\left(x_{2}\right) \\
& \quad=\frac{1}{(2 \pi)^{4}} \int \frac{d k\left[K\left(-k^{2} l^{2}\right)\right]^{2}}{k^{2}+m^{2}} e^{i k x}=\frac{1}{(2 \pi)^{4}} \int d k \tilde{D}\left(k^{2}\right) e^{i k x}
\end{aligned}
$$

where all integrations are performed over the Euclidean $k$-space. The function $D(x)$ satisfies the conditions

(a) $D(x)$ is real and positive,

(b) $D(0)<\infty$,

(c) $\tilde{D}\left(k^{2}\right) \geqq 0$. 
The $S$-matrix in the representation (1.3) contains volume divergences connected with the translation invariance of our theory. Therefore we have to introduce the integration over a finite volume $V$ in (1.3). We will consider that it is a sphere of the radius $L$ so that $V=\frac{1}{2} \pi^{2} L^{4}$.

Besides it is convenient to introduce the function

$$
\mathscr{D}(x)=\frac{1}{(2 \pi)^{4}} \int d k \tilde{\mathscr{D}}\left(k^{2}\right) e^{i k x}
$$

such that

$$
D\left(x_{1}-x_{2}\right)=\int d y \mathscr{D}\left(x_{1}-y\right) \mathscr{D}\left(y-x_{2}\right) .
$$

Let us define the function

$$
D_{V}\left(x_{1}, x_{2}\right)=\int_{V} d y \mathscr{D}\left(x_{1}-y\right) \mathscr{D}\left(y-x_{2}\right) .
$$

When $V \rightarrow \infty$ we obtain

$$
D_{V}\left(x_{1}, x_{2}\right)=D\left(x_{1}-x_{2}\right)+O\left(\exp \left\{- \text { const } V^{1 / 4}\right\}\right)
$$

because $D(x) \sim e^{-m \sqrt{x^{2}}}$ for $\quad x^{2} \rightarrow \infty$.

Then the regularized $S$-matrix can be represented in the form

$$
\begin{aligned}
S_{V}[g, \varphi]= & \exp \left\{\frac{1}{2} \int_{V} \int_{V} d x_{1} d x_{2} D_{V}\left(x_{1}, x_{2}\right) \frac{\delta^{2}}{\delta \varphi\left(x_{1}\right) \delta \varphi\left(x_{2}\right)}\right\} \\
& \cdot \exp \left\{g \int_{V} d x U(\varphi(x))\right\}
\end{aligned}
$$

The perturbation series for the $S$-matrix (1.9) can be written

$$
\begin{aligned}
S_{V}[g, \varphi]= & \sum_{n=0}^{\infty} \frac{g^{n}}{n !} \int_{V} d x_{1} \cdot \ldots \cdot \int_{V} d x_{n} \\
& \cdot \int_{-\infty}^{\infty} d \beta_{1} \tilde{U}\left(\beta_{1}\right) e^{i \beta_{1} \varphi\left(x_{1}\right)} \cdot \ldots \cdot \int_{-\infty}^{\infty} d \beta_{n} \tilde{U}\left(\beta_{n}\right) e^{i \beta_{n} \varphi\left(x_{n}\right)} \\
& \cdot \exp \left\{-\frac{1}{2} \sum_{i, j-1}^{n} \beta_{i} D_{V}\left(x_{i}, x_{j}\right) \beta_{j}\right\}
\end{aligned}
$$

Having introduced the notations

$$
\begin{aligned}
\int_{V} d \mu & =\int_{V} d x \int_{-\infty}^{\infty} d \beta \tilde{U}(\beta) e^{-\frac{1}{2} \beta^{2} D_{V}(x, x)} \cdot e^{i \beta \varphi(x)}, \\
w_{i j} & =w_{V}\left(\beta_{i} \beta_{j} ; x_{i}, x_{j}\right)=\exp \left\{-\beta_{i} \beta_{j} D_{V}\left(x_{i}, x_{j}\right)\right\}-1
\end{aligned}
$$

the series (1.10) can be rewritten in the compact form

$$
S_{V}[g, \varphi]=\sum_{n=0}^{\infty} \frac{g^{n}}{n !} \int_{V} d \mu_{1} \cdot \ldots \cdot \int_{V} d \mu_{n} \prod_{1 \leqq i<j \leqq n}\left(1+w_{i j}\right) .
$$


The product $\prod\left(1+w_{i j}\right)$ in (1.12) can be interpreted as a sum of all possible connected and disconnected ordinary diagrams with $n$ vertices when the function $w_{i j}$ corresponds to the edge of the diagram joining the points $i$ and $j$.

In the representation

$$
S_{V}[g, \varphi]=\exp \left\{B_{V}[g, \varphi]\right\}
$$

the functional $B_{V}[g, \varphi]$ contains the connected diagrams only

$$
\begin{aligned}
B_{V}[g, \varphi] & =\sum_{n=1}^{\infty} \frac{g^{n}}{n !} \int d \mu_{1} \cdot \ldots \cdot \int d \mu_{n}\left\{\prod_{1 \leqq i<j \leqq n}\left(1+w_{i j}\right)\right\}_{c} \\
& =\sum_{m=0}^{\infty} \frac{1}{m !} \int d x_{1} \cdot \ldots \cdot \int d x_{m} B_{m}\left(V ; x_{1}, \ldots, x_{m}\right) \varphi\left(x_{1}\right) \cdot \ldots \cdot \varphi\left(x_{m}\right)
\end{aligned}
$$

where $\left\{\Pi\left(1+w_{i j}\right)\right\}_{c}$ means that we have to keep only the connected diagrams from all the product.

The vacuum energy is defined by the expression

$$
\begin{aligned}
E(g)= & -\lim _{V \rightarrow \infty} \frac{1}{V} \ln S_{V}[g, 0]=-\lim _{V \rightarrow \infty} \frac{1}{V} B_{V}[g, 0] \\
= & -\sum_{n=1}^{\infty} \frac{g^{n}}{n !} \int d \mu_{1} \cdot \ldots \cdot \int d \mu_{n} n^{4} \delta\left(x_{1}+\ldots+x_{n}\right) \\
& \cdot\left\{\prod_{i<j}\left(1+w\left(\beta_{i} \beta_{j}, x_{i}-x_{j}\right)\right)\right\}_{c} .
\end{aligned}
$$

Now let us consider a physical process of the interaction of $m$ scalar particles. Then the connected part of the amplitude of such a process is defined in the Euclidean region of momenta variables by the following expression:

$$
\begin{aligned}
\tilde{B}_{m}\left(p_{1}, \ldots, p_{m}\right) & =\int d x_{1} \cdot \ldots \cdot \int d x_{m} e^{i\left(p_{1} x_{1}+\ldots+p_{m} x_{m}\right)} \lim _{V \rightarrow \infty} B_{m}\left(V ; x_{1}, \ldots, x_{m}\right), \\
\tilde{B}_{m}\left(p_{1}, \ldots, p_{m}\right) & =(2 \pi)^{4} \delta\left(p_{1}+\ldots+p_{m}\right) T_{m}\left(p_{1}, \ldots, p_{m}\right), \\
T_{m}\left(p_{1}, \ldots, p_{m}\right) & =\int d x_{1} \cdot \ldots \cdot \int d x_{m} \cdot m^{4} \delta\left(x_{1}+\ldots+x_{m}\right) e^{i p_{j} x_{j}} \lim _{V \rightarrow \infty} B_{m}\left(V ; x_{1}, \ldots, x_{m}\right) .
\end{aligned}
$$

In the perturbation theory we obtain

$$
\begin{aligned}
& T_{m}\left(p_{1}, \ldots, p_{m}\right)=\sum_{n=1}^{\infty} \frac{g^{n}}{n !} \int_{-\infty}^{\infty} d \beta_{1} \tilde{U}\left(\beta_{1}\right) e^{-\frac{1}{2} \beta_{1}^{2} D(0)} \cdot \ldots \\
& \cdot \int_{-\infty}^{\infty} d \beta_{n} \tilde{U}\left(\beta_{n}\right) e^{-\frac{1}{2} \beta_{n}^{2} D(0)} \cdot \int d x_{1} \cdot \ldots \cdot \int d x_{n} n^{4} \delta\left(x_{1}+\ldots+x_{n}\right) \\
& \cdot\left\{\prod_{i<j}\left(1+w_{i j}\left(\beta_{i} \beta_{j}, x_{i}-x_{j}\right)\right)\right\} \prod_{c} \prod_{k=1}^{n}\left(\sum_{j=1}^{n} \beta_{j} e^{i p_{k} x_{j}}\right) .
\end{aligned}
$$

In papers $[2,3]$ it was proved that in the class of theories under consideration the perturbation series (1.15) and (1.17) converge in the Euclidean region of momenta variables $\left(p_{1}, \ldots, p_{n}\right)$ for

$$
|g|<g_{0}
$$

where $g_{0}$ depends on the parameters of our theory and is essentially defined by two conditions (1.2) and (1.5), i.e. $D(0)<\infty$ and $\max |V(\varphi)|=1$. 


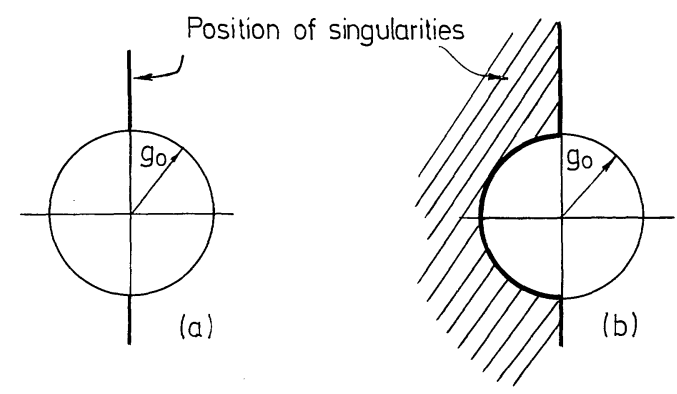

Fig. 1a and b. The position of singularities in the complex $g$-plane for add (a) and even (b) functions $U(\varphi)$

Our aim is to find the location of singularities of amplitudes in the complex $g$ plane and to do analytical continuation to the region $|g|>g_{0}$.

We will prove that the singularities of amplitudes are on the imaginary axis in the $g$-plane for the odd interaction functions $U(\varphi)$ (Fig. 1a) and in the left part of $g$ plane for the even interaction functions $U(\varphi)$ (Fig. 1b).

Our proof is the following:

(1) we obtain the representation for the $S$-matrix (1.10) in the form of a functional integral and prove that this integral exists for any finite $V$;

(2) we introduce the function $F_{V}(\zeta, \varphi)=S_{V}\left[\frac{\zeta}{V}, \varphi\right]$ and prove that this function is an entire analytical function of the first order in complex $\zeta$-plane;

(3) the Hadamard representation in the form of infinite product with respect to zeroes of an entire function is written down for the function $F_{V}(\zeta, \varphi)$ and the functional $B_{V}=\ln S_{V}$ is found in this new representation;

(4) we prove that there exist different limits when $V \rightarrow \infty$ for the zeroes of entire function $F_{V}(\zeta, \varphi)$ making use of the Jensen and Carleman formulas;

(5) the obtained limiting correlations together with the results of perturbation theory give new representations for physical amplitudes under consideration for any $g$; $p^{2}$.

(6) the two-point Green function is continued in the physical region of momenta

\section{Representation of the $S$-Matrix in the Form of a Functional Integral}

Let us consider the functional integral

$$
\begin{aligned}
S_{V}[g, \varphi] & =\int \delta \Lambda \exp \left\{-\frac{1}{2} \int_{V} d x \Lambda^{2}(x)+g \int_{V} d x U\left(\varphi(x)+\int_{V} d y \mathscr{D}(x-y) \Lambda(y)\right)\right\} \\
& =\int \delta \Lambda \exp \left\{-\frac{1}{2} \int_{V} \Lambda^{2}+g \int_{V} U\left(\varphi+\int_{V} \mathscr{D} \Lambda\right)\right\} .
\end{aligned}
$$

Here the integration over $x$ is carried out over a four-dimensional sphere of the volume $V$. The normalization is chosen in such a way that

$$
S_{V}[0, \varphi]=\int \delta \Lambda \exp \left\{-\frac{1}{2} \int_{V} \Lambda^{2}\right\}=1 \text {. }
$$


The function $\mathscr{D}(x)$ is defined in (1.6) and (1.7).

The functional integral in (2.1) will be defined in the following way. Let the system of functions $\left\{g_{s}(x), s=0,1,2, \ldots\right\}$ be complete orthonormal in the volume $V$ :

$$
\begin{aligned}
& \int_{V} d x g_{s}(x) g_{s^{\prime}}(x)=\delta_{s s^{\prime}} \\
& \sum_{s=0}^{\infty} g_{s}(x) g_{s}\left(x^{\prime}\right)=\delta\left(x-x^{\prime}\right) .
\end{aligned}
$$

Then the function $\Lambda(x)$ can be represented in the form

$$
\Lambda(x)=\sum_{s=0}^{\infty} u_{s} g_{s}(x)
$$

from which

$$
\begin{aligned}
& \int_{V} d x \Lambda^{2}(x)=\sum_{s=0}^{\infty} u_{s}^{2}, \\
& \int_{V} d y \mathscr{D}(x-y) \Lambda(y)=\sum_{s=0}^{\infty} u_{s} \mathscr{D}_{s}(x)
\end{aligned}
$$

where

$$
\mathscr{D}_{s}(x)=\int_{V} d y \mathscr{D}(x-y) g_{s}(y) \text {. }
$$

So far as the function $\mathscr{D}(x)$ is smooth

$$
\lim _{s \rightarrow \infty}\left|\mathscr{D}_{s}(x)\right|=0
$$

uniformly for all $x \in V$ and

$$
\sum_{s=0}^{\infty} \mathscr{D}_{s}^{2}(x)=D(0)<\infty
$$

The differential $\delta \Lambda$ in $(2.1)$ is defined as

$$
\int \delta \Lambda=\prod_{s=0}^{\infty} \int_{-\infty}^{\infty} \frac{d u_{s}}{\sqrt{2 \pi}} .
$$

Then the functional integral (2.1) will be defined as the limit

$$
\begin{aligned}
S_{V}[g, \varphi]= & \lim _{N \rightarrow \infty} S_{V}^{(N)}[g, \varphi], \\
S_{V}^{(N)}[g, \varphi]= & \prod_{s=0}^{N} \int_{-\infty}^{\infty} \frac{d u_{s}}{\sqrt{2 \pi}} \\
& \cdot \exp \left\{-\frac{1}{2} \sum_{s=0}^{N} u_{s}^{2}+g \int_{V} d x U\left(\varphi(x)+\sum_{s=0}^{N} u_{s} \mathscr{D}_{s}(x)\right)\right\} .
\end{aligned}
$$


The normalization is the following:

$$
S_{V}^{(N)}[0, \varphi]=\prod_{s=0}^{N} \int_{-\infty}^{\infty} \frac{d u_{s}}{\sqrt{2 \pi}} \exp \left\{-\frac{1}{2} \sum_{s=0}^{N} u_{s}^{2}\right\}=1 .
$$

Let us prove the existance of the limit in (2.8). We consider the difference

$$
\begin{aligned}
\Delta_{N}^{N+M} & =S_{V}^{(N+M)}[g, \varphi]-S_{V}^{(N)}[g, \varphi] \\
& =\int_{N+M}\left[\exp \left\{g \int U\left(\varphi+\begin{array}{c}
N+M \\
\sigma \\
0
\end{array}\right)\right\}-\exp \left\{g \int U\left(\varphi+\begin{array}{r}
N \\
0
\end{array}\right)\right\}\right]
\end{aligned}
$$

with notations

$$
\begin{aligned}
& \int_{N}=\prod_{s=0}^{N} \int_{-\infty}^{\infty} \frac{d u_{s}}{\sqrt{2 \pi}} \exp \left\{-\frac{1}{2} \sum_{s=0}^{N} u_{s}^{2}\right\}, \\
& \sigma=N_{N_{1}}^{N_{2}}(x)=\sum_{s=N_{1}}^{N_{2}} u_{s} \mathscr{D}_{s}(x) .
\end{aligned}
$$

After simple transformations one can obtain

$$
\begin{aligned}
& \Delta_{N}^{N+M}=g \int_{0}^{1} d \lambda \int_{N+M} \int_{V} d x \underset{N+1}{N+M} \sigma(x) U^{\prime}(\varphi(x)+\underset{0}{\sigma}(x)+\lambda \underset{N+1}{\sigma+M}(x)) \\
& \cdot \exp \left\{g \int_{V} U\left(\varphi+\begin{array}{cc}
N & \\
\sigma & N+M \\
0 & \sigma \\
N+1
\end{array}\right)\right\} \\
& =\frac{g}{2} \int_{0}^{1} d \lambda \lambda \int_{-1}^{1} d v \int_{N+M} \\
& \cdot\left\{\int_{V} d x\left(\begin{array}{c}
N+M \\
\sigma \\
N+1
\end{array}(x)\right)^{2} U^{\prime \prime}(\varphi(x)+\underset{0}{\sigma} \sigma(x)+\lambda v \underset{N+1}{\sigma+M}(x))\right. \\
& \left.+g\left[\int_{V} d x \underset{N+1}{N+M}(x) U^{\prime}\left(\varphi+\begin{array}{cc}
N \\
\sigma \\
0
\end{array} \quad \begin{array}{c}
N+M \\
N+1
\end{array}\right)\right]^{2}\right\} \\
& \cdot \exp \left\{g \int_{V} U\left(\varphi+\begin{array}{c}
N \\
\sigma \\
0
\end{array}+\lambda \nu \begin{array}{c}
N+M \\
N+1
\end{array}\right)\right\} \text {. }
\end{aligned}
$$

The following estimation can be obtained using the obvious inequalities

$$
\begin{aligned}
& \left|\Delta_{N}^{N+M}\right| \leqq \frac{|g| V}{4} e^{|g| V} \\
& \cdot\left\{\max _{u}\left|U^{\prime \prime}(u)\right|+|g| V\left(\max _{u}\left|U^{\prime}(u)\right|\right)^{2}\right\} \frac{1}{V} \int_{V} d x \sum_{s=N+1}^{\infty} \mathscr{D}_{s}^{2}(x) .
\end{aligned}
$$

According to (2.6) and due to the convergence of the series $\sum_{s} \mathscr{D}_{s}^{2}(x)$ the right hand side of (2.13) can be made arbitrary small when $N \rightarrow \infty$. It means that for any $\varepsilon>0$ there exists a number $N_{0}$ that for all $M>0$ and $N>N_{0}$

$$
\left|S_{V}^{(N+M)}[g, \varphi]-S_{V}^{(N)}[g, \varphi]\right|<\varepsilon
$$


i.e. the sequence $S_{V}^{(N)}[g, \varphi]$ is fundamental. Further, one can obtain easily the following estimation for any $N>0$ from the representation (2.9)

$$
\left|S_{V}^{(N)}[g, \varphi]\right| \leqq e^{|g| V} .
$$

Thus, the limit in (2.8) does exist and does not depend on the choice of the basis $\left\{g_{s}\right\}$. This limit defines the functional integral (2.1).

Now let us obtain the perturbation series from the representation (2.8). We have

$$
\begin{aligned}
S_{V}[g, \varphi]= & \lim _{N \rightarrow \infty} \prod_{s=0}^{N} \int_{-\infty}^{\infty} \frac{d u_{s}}{\sqrt{2 \pi}} \exp \left\{-\frac{1}{2} \sum_{s=0}^{N} u_{s}^{2}\right\} \\
& \cdot \sum_{n=0}^{\infty} \frac{g^{n}}{n !} \prod_{j=1}^{n} \int_{V} d x_{j} \int_{-\infty}^{\infty} d \beta_{j} \tilde{U}\left(\beta_{j}\right) e^{i \beta_{j} \varphi\left(x_{j}\right)} \exp \left\{i \beta_{j} \sum_{s=0}^{N} u_{s} \mathscr{D}_{s}\left(x_{j}\right)\right\} \\
= & \sum_{n=0}^{\infty} \frac{g^{n}}{n !} \prod_{j=1}^{n} \int_{V} d x_{j} \int_{-\infty}^{\infty} d \beta_{j} \tilde{U}\left(\beta_{j}\right) e^{i \beta_{j} \varphi\left(x_{j}\right)} \\
& \cdot \lim _{N \rightarrow \infty} \exp \left\{-\frac{1}{2} \sum_{s=0}^{N}\left(\sum_{k=1}^{n} \beta_{k} \mathscr{D}_{s}\left(x_{k}\right)\right)^{2}\right\}
\end{aligned}
$$

In the limit $N \rightarrow \infty$ we have

$$
\begin{aligned}
\lim _{N \rightarrow \infty} & \sum_{s=0}^{N}\left(\sum_{k=1}^{n} \beta_{k} \mathscr{D}_{s}\left(x_{k}\right)\right)^{2} \\
\quad= & \sum_{i, j=1}^{n} \beta_{i} \beta_{j} \lim _{N \rightarrow \infty} \sum_{s=0}^{N} \mathscr{D}_{s}\left(x_{i}\right) \mathscr{D}_{s}\left(x_{j}\right) \\
\quad= & \sum_{i, j=1}^{n} \beta_{i} \beta_{j} \int_{V} d y \mathscr{D}\left(x_{i}-y\right) \mathscr{D}\left(y-x_{j}\right)=\sum_{i, j=1}^{n} \beta_{i} D_{V}\left(x_{i}, x_{j}\right) \beta_{j} .
\end{aligned}
$$

Finally from (2.16) we obtain the representation (1.10).

\section{Properties of the $S$-Matrix in the Finite Volume}

In the previous section we obtained the representation of the functional integral (2.1) which is well defined. In this section we will investigate in detail the properties of the $S$-matrix in the complex $g$-plane using the representation (2.1).

Let us introduce the following function of complex variable $\zeta$ :

$$
\begin{aligned}
F_{V}(\zeta, \varphi) & =S_{V}\left[\frac{\zeta}{V}, \varphi\right] \\
& =\int \delta \Lambda \exp \left\{-\frac{1}{2} \int_{V} \Lambda^{2}+\zeta \frac{1}{V} \int_{V} U\left(\varphi+\int_{V} \mathscr{D} \Lambda\right)\right\}
\end{aligned}
$$

The function $F_{V}(\zeta, \varphi)$ is an entire analytical function of the first order in the $\zeta$-plane because the integral (3.1) exists for any complex $\zeta=\xi+i \eta$ and the following 
estimation is valid:

$$
\begin{aligned}
& \left|F_{V}(\zeta, \varphi)\right| \leqq \int \delta \Lambda \exp \left\{-\frac{1}{2} \int_{V} \Lambda^{2}+\xi \frac{1}{V} \int_{V} U\left(\varphi+\int_{V} \mathscr{D} \Lambda\right)\right\} \\
& \leqq \frac{1}{V} \int_{V} d x \int \delta \Lambda \exp \left\{-\frac{1}{2} \int_{V} \Lambda^{2}+\xi U\left(\varphi(x)+\int_{V} d y \mathscr{D}(x-y) \Lambda(y)\right)\right\} \\
& =\frac{1}{V} \int_{V} d x \int_{-\infty}^{\infty} \frac{d \Lambda}{\sqrt{2 \pi}} \exp \left\{-\frac{1}{2} \Lambda^{2}+\xi U\left(\varphi(x)+\Lambda \sqrt{\left.D_{V}(x, x)\right)}\right\} \leqq e^{|\xi|}\right.
\end{aligned}
$$

In order to obtain the estimation (3.2) we make use of the inequality

$$
h\left(\frac{1}{V} \int_{V} d x \Psi(x)\right) \leqq \frac{1}{V} \int_{V} d x h(\Psi(x))
$$

which is valid for any convex downwards functions $\left(h^{\prime \prime}(s) \geqq 0\right)$ and any real functions $\Psi(x)$ (see [4]).

One can see that this estimation (3.2) does not depend on the volume $V$.

Besides the function $F_{V}(\zeta, \varphi)$ is analytic with respect to $\varphi$ in a vicinity of the point $\varphi=0$. It should be noted that

$$
\lim _{V \rightarrow \infty} \frac{1}{V} \int_{V} d x e^{i \xi \varphi(x)}=1
$$

if $\int d x \varphi^{n}(x)<\infty$ for any $n>0$, and this limit does not depend on $\varphi(x)$. We are interested in the coefficients of the $S$-matrix expansion in the vicinity of the point $\varphi(x)=0$ because these coefficient functions determine any matrix elements of different physical processes. Therefore we shall estimate all limits $V \rightarrow \infty$ for $\varphi(x)=\varphi=$ const where $\varphi$ is sufficiently small.

Consider the function

$$
F_{V}(\zeta, \varphi)=\int \delta \Lambda \exp \left\{-\frac{1}{2} \int_{V} \Lambda^{2}+\zeta \frac{1}{V} \int_{V} U\left(\varphi+\int_{V} \mathscr{D} \Lambda\right)\right\} .
$$

Because $F_{V}(\zeta, \varphi)$ is an entire function of the first order the Paley-Wiener representation is valid for it:

$$
F_{V}(\zeta, \varphi)=\int_{-1}^{1} d t \phi_{V}(t, \varphi) e^{\zeta t}
$$

for the odd interaction functions $U(\varphi)=-U(-\varphi)$ and

$$
F_{V}(\zeta, \varphi)=\int_{0}^{1} d t \Psi_{V}(t, \varphi) e^{\zeta t}
$$

for the even functions $U(\varphi)=U(-\varphi)$. The functions $\phi_{V}$ and $\Psi_{V}$ are defined by the integral

$$
\int \delta \Lambda e^{-\frac{1}{2} \int \Lambda^{2}} \delta\left(t-\frac{1}{V} \int_{V} U\left(\varphi+\int_{V} \mathscr{D} \Lambda\right)\right) .
$$


The functions $\phi_{V}(t, \varphi)$ and $\Psi_{V}(t, \varphi)$ are summable functions for which the following correlations are valid:

$$
\begin{aligned}
& \phi_{V}(t, \varphi) \geqq 0, \Psi_{V}(t, \varphi) \geqq 0, \\
& \phi_{V}( \pm 1, \varphi)=\Psi_{V}(0, \varphi)=\Psi_{V}(1, \varphi)=0, \\
& \int_{-1}^{1} d t \phi_{V}(t, \varphi)=\int_{0}^{1} d t \Psi_{V}(t, \varphi)=1 .
\end{aligned}
$$

It follows from the representation (3.5) that for $\zeta=\xi+i \eta, \xi>0$ and $|\zeta| \rightarrow \infty$

$$
F_{V}(\zeta, \varphi)=O\left(\frac{1}{\zeta} e^{\zeta}\right)
$$

Now let us obtain the behaviour of the function $\phi_{V}(t)=\phi_{V}(t, 0)$ near the point $t=+1$. We consider the expression

$$
\phi_{V}(1-\tau)=\int \delta \Lambda e^{-\frac{1}{2} \int \Lambda^{2}} \delta\left(\tau-\frac{1}{V} \int_{V}\left[U\left(\varphi_{0}\right)-U\left(\int_{V} \mathscr{D} \Lambda\right)\right]\right)
$$

where the point $\varphi_{0}$ defines the maximum of the function $U(\varphi)$, i.e. $U\left(\varphi_{0}\right)=1$. Let $\tau \rightarrow 0$, then

$$
\begin{aligned}
& \phi_{V}(1-\tau) \simeq \int \delta \Lambda \exp \left\{-\frac{1}{2} \int_{V} \Lambda^{2}\right\} \\
& \cdot \delta\left(\tau-\left|U^{\prime \prime}\left(\varphi_{0}\right)\right| \frac{1}{V} \int d x\left(\varphi_{0}-\int_{V} d y \mathscr{D}(x-y) \Lambda(y)\right)^{2}\right) .
\end{aligned}
$$

This functional integral can be calculated in the explicit form for the sufficiently large volume $V$ :

$$
\phi_{V}(1-\tau) \simeq \frac{1}{2 \pi} \int_{-\infty}^{\infty} \frac{d v \exp \left\{i v \tau-i v a \varphi_{0}^{2}\left[1+\frac{2 i}{V} v a \tilde{D}\right]^{-1}\right\}}{\left[1+\frac{2}{V} i v a \tilde{D}\right]^{V / 2}}
$$

where

$$
a=\left|U^{\prime \prime}\left(\varphi_{0}\right)\right|, \tilde{D}=\int d x D(x) .
$$

When $\tau \rightarrow 0$ this function tends to zero as

$$
\phi_{V}(1-\tau)=O\left(\tau^{V / 2}\right) \text {. }
$$

Therefore the function $\phi_{V}(t)$ is $\left[\frac{V}{2}\right]$ times differentiable and for $|\zeta| \rightarrow \infty$

$$
F_{V}(\zeta, 0)=\int_{-1}^{1} d t \phi_{V}(t) e^{\zeta t}=O\left(\frac{\operatorname{ch} \zeta}{\zeta^{V / 2}}\right)
$$

Let us calculate the behaviour of the ratio

$$
F_{V}^{\prime}(\xi) / F_{V}(\xi)
$$


for $\xi \rightarrow \infty$ and any $V$, where the function $F_{V}(\xi)=F_{V}(\xi, 0)$ is defined by (3.4). For $F_{V}^{\prime}(\xi)$ we have

$$
\begin{aligned}
F_{V}^{\prime}(\xi)= & \int \delta \Lambda \frac{1}{V} \int_{V} U\left(\int_{V} \mathscr{D} \Lambda\right) \\
& \cdot \exp \left\{-\frac{1}{2} \int_{V} \Lambda^{2}+\xi \frac{1}{V} \int_{V} U\left(\int_{V} \mathscr{D} \Lambda\right)\right\} .
\end{aligned}
$$

The function $F_{V}(\xi)$ can be represented in the form

$$
F_{V}(\xi)=e^{\xi} f_{V}(\xi)
$$

where

$$
f_{V}(\xi)=\int \delta \Lambda \exp \left\{-\frac{1}{2} \int_{V} \Lambda^{2}-\xi \frac{1}{V} \int_{V}\left[1-U\left(\int_{V} \mathscr{D} \Lambda\right)\right]\right\} .
$$

Making use of the inequality (3.3) we obtain

$$
f_{V}(\xi) \leqq \int_{-\infty}^{\infty} \frac{d \Lambda}{\sqrt{2 \pi}} \exp \left\{-\frac{1}{2} \Lambda^{2}-\xi[1-U(\Lambda \sqrt{D})]\right\}
$$

from which

$$
f_{V}(\xi) \leqq O\left(\frac{1}{\sqrt{\xi}}\right) \quad(\xi \rightarrow+\infty)
$$

This estimation is too low in contrast with (3.12) but it is sufficient for our aim.

The function $F_{V}^{\prime}(\xi)$ can be represented in the following form

$$
F_{V}^{\prime}(\xi)=e^{\xi}\left[f_{V}(\xi)-\Psi_{V}(\xi)\right]
$$

where

$$
\begin{aligned}
\Psi_{V}(\xi)= & \int \delta \Lambda \frac{1}{V} \int_{V}\left[1-U\left(\int_{V} \mathscr{D} \Lambda\right)\right] \\
& \cdot \exp \left\{-\frac{1}{2} \int_{V} \Lambda^{2}-\xi \frac{1}{V} \int_{V}\left[1-U\left(\int_{V} \mathscr{D} \Lambda\right)\right]\right\} .
\end{aligned}
$$

Consider the function $h(u)=u e^{-u}$, for $u \geqq 2$ we have $h^{\prime \prime}(u)=(u-2) e^{-u} \geqq 0$.

The function $\Psi_{\nu}(\xi)$ can be transformed

$$
\Psi_{V}(\xi)=\frac{1}{\xi}\left[\Psi_{1 V}(\xi)-2 f_{V}(\xi)\right]
$$

where $f_{V}(\xi)$ is defined by (3.14) and

$$
\Psi_{1 V}(\xi)=\int \delta \Lambda e^{-\frac{1}{2} \int A_{V}^{2}} h\left(\xi \frac{1}{V} \int_{V}\left[1+\frac{2}{\xi}-U\left(\int_{V} \mathscr{D} A\right)\right]\right) .
$$


Making use of the inequality (3.3) and

$$
\xi \frac{1}{V} \int_{V}\left[1+\frac{2}{\xi}-U\left(\int_{V} \mathscr{D} \Lambda\right)\right] \geqq 2
$$

we can obtain the following estimation

$$
\begin{aligned}
\left|\Psi_{1 V}(\xi)\right| \leqq & \frac{1}{V} \int_{V} d x \int \delta \Lambda e^{-\frac{1}{2} \int \Lambda^{2}} h\left(\xi\left[1+\frac{2}{\xi}-U\left(\int_{V} d y \mathscr{D}(x-y) \Lambda(y)\right)\right]\right) \\
= & \xi \int_{-\infty}^{\infty} \frac{d \Lambda}{\sqrt{2 \pi}} e^{-\frac{1}{2} \Lambda^{2}\left[1+\frac{2}{\xi}-U(\Lambda \sqrt{D})\right]} \\
& \cdot \exp \{-\xi[1-U(\Lambda \sqrt{D})]+2\}=O\left(\frac{1}{\sqrt{\xi}}\right) \quad(\xi \rightarrow \infty)
\end{aligned}
$$

from which

$$
\Psi_{V}(\xi)=O\left(\frac{1}{\xi^{3 / 2}}\right) \quad(\xi \rightarrow+\infty) .
$$

Finally we have for $F_{V}^{\prime}(\xi)$ when $\xi \rightarrow+\infty$

$$
F_{V}^{\prime}(\xi)=e^{\xi} f_{V}(\xi)\left[1+O\left(\frac{1}{\xi}\right)\right]
$$

Then we obtain for the ratio

$$
\frac{F_{V}^{\prime}(\xi)}{F_{V}(\xi)}=1+O\left(\frac{1}{\xi}\right)
$$

when $\xi \rightarrow+\infty$. It should be noted that this estimation is valid for any $V$.

Now let us obtain the estimation for $F_{V}(\zeta, \varphi)$ when $\zeta$ is fixed and $V \rightarrow \infty$. For this aim we consider the perturbation series (2.18). We represent this series in the form

$$
\begin{aligned}
F_{V}(\zeta, \varphi)= & \sum_{n=0}^{\infty} \frac{\zeta^{n}}{n !} \prod_{j=1}^{n}\left\{\frac{1}{V} \int_{V} d \mu_{j} \exp \left(-\frac{1}{2} \beta_{j}^{2} D_{V}\left(x_{j}, x_{j}\right)\right)\right\} \\
& \cdot \exp \left\{-\sum_{i<j} \beta_{i} D_{V}\left(x_{i}-x_{j}\right) \beta_{j}\right\}
\end{aligned}
$$

Making use of the identity

$$
e^{-\Delta}=1+\int_{0}^{1} d \lambda \Delta e^{-\lambda \Delta}
$$

where $\Delta=\sum_{i<j} \beta_{i} D_{V}\left(x_{i}, x_{j}\right) \beta_{j}$ the function $F_{V}(\zeta, \varphi)$ can be written as

$$
F_{V}(\zeta, \varphi)=\sum_{n=0}^{\infty} \frac{\zeta^{n}}{n !}\left\{\frac{1}{V} \int_{V} d x U_{0}(\varphi(x))\right\}^{n}+Q_{V}(\zeta, \varphi)
$$


where

$$
\begin{aligned}
Q_{V}(\zeta, \varphi)= & \zeta^{2} \frac{1}{V^{2}} \int_{V} d x_{1} \int_{V} d x_{2} D_{V}\left(x_{1}, x_{2}\right) \\
& \cdot \int_{0}^{1} d \lambda \int \delta \Lambda \exp \left\{-\frac{1}{2} \int_{V} \Lambda^{2}+\zeta \frac{1}{V} \int_{V} U_{\lambda}\left(\varphi+\sqrt{\lambda} \int_{V} \mathscr{D} \Lambda\right)\right\} \\
& \cdot U_{\lambda}^{\prime}\left(\varphi\left(x_{1}\right)+\sqrt{\lambda} \int_{V} d y \mathscr{D}\left(x_{1}-y\right) \Lambda(y)\right) \\
& \cdot U_{\lambda}^{\prime}\left(\varphi\left(x_{2}\right)+\sqrt{\lambda} \int_{V} d y \mathscr{D}\left(x_{2}-y\right) \Lambda(y)\right) .
\end{aligned}
$$

Here $(u=0, \lambda)$

$$
U_{u}(\varphi)=\int_{-\infty}^{\infty} d \beta \tilde{U}(\beta) \exp \left\{-\frac{1-u}{2} \beta^{2} D_{V}(x, x)+i \beta \varphi(x)\right\} .
$$

In the limit $V \rightarrow \infty$ for $Q_{V}$ we obtain the estimation

$$
\left|Q_{V}(\zeta, \varphi)\right| \leqq \frac{|\zeta|^{2}}{V} \int d x D(x) \int_{0}^{1} d \lambda \max _{u}\left|U^{\prime}(u)\right| e^{\sqrt{\lambda} \mathrm{Re} \zeta}
$$

from which

$$
\lim _{V \rightarrow \infty} Q_{V}(\zeta, \varphi)=0
$$

Then we have

$$
\begin{aligned}
F(\zeta, \varphi) & =\lim _{V \rightarrow \infty} F_{V}(\zeta, \varphi) \\
& =\exp \left\{\zeta \int_{-\infty}^{\infty} d \beta \tilde{U}(\beta) \exp \left[-\frac{1}{2} \beta^{2} D(0)+i \beta \varphi\right]\right\}
\end{aligned}
$$

where $\varphi=$ const as it was mentioned above. The limiting function $F(\zeta, \varphi)$ is an entire analytical function of the first order in the complex $\zeta$-plane.

\section{Hadamard Representation}

The function $F_{V}(\zeta, \varphi)$ in (3.1) is an entire function of the first order in the $\zeta$-plane and it is real for real $\zeta=\xi$. Therefore the Hadamard representation is valid for it (see, for example [5]):

$$
F_{V}(\zeta, \varphi)=e^{a(V, \varphi) \zeta} \prod_{j=1}^{\infty}\left(1-\frac{\zeta}{a_{j}(V, \varphi)}\right) e^{\frac{\zeta}{a_{j}(V, \varphi)}}\left(1-\frac{\zeta}{a_{j}^{*}(V, \varphi)}\right) e^{\frac{\zeta}{a_{j}^{\frac{\zeta}{j}(V, \varphi)}}}
$$

Here $a_{j}(V, \varphi)(j=1,2, \ldots)$ are zeroes of our function $F_{V}(\zeta, \varphi)$. Let us denote

$$
\begin{aligned}
a_{j}(V, \varphi) & =r_{j}(V, \varphi) e^{i \theta_{j}(V, \varphi)}, \quad(j=1,2, \ldots) \\
0 & \leqq \theta_{j}(V, \varphi) \leqq \pi
\end{aligned}
$$


Every zero $a_{j}(V, \varphi)$ is an analytic functional of the field $\varphi(x)$ at the point $\varphi=0$. Let us represent this functional $a_{j}(V, \varphi)$ in the form of functional decomposition

$$
\begin{aligned}
& a_{j}(V, \varphi)=a_{j}(V) \\
& \quad+\sum_{m=1}^{\infty} \frac{1}{m !} \int d x_{1} \ldots \int d x_{m} a_{j}^{(m)}\left(V ; x_{1}, \ldots, x_{m}\right) \varphi\left(x_{1}\right) \ldots \varphi\left(x_{m}\right) .
\end{aligned}
$$

Then the following representation for the functional $B_{V}[g, \varphi](1.13)$, describing connected diagrams only, is valid

$$
\begin{aligned}
B_{V}[g, \varphi] & =\ln F_{V}[g V, \varphi] \\
& =g V a(V, \varphi)-\sum_{n=2}^{\infty} \frac{g^{n}}{n} 2 \operatorname{Re} \sum_{j=1}^{\infty}\left[\frac{V}{a_{j}(V, \varphi)}\right]^{n} .
\end{aligned}
$$

The comparison with the perturbation series gives

$$
a(V, \varphi)=\frac{1}{V} \int_{V} d x \int_{-\infty}^{\infty} d \beta \tilde{U}(\beta) \exp \left\{-\frac{1}{2} \beta^{2} D_{V}(x, x)+i \beta \varphi(x)\right\} .
$$

Now let us obtain in the framework of the representation (4.4) the expressions for the vacuum energy $E(g)$ in (1.15) and the amplitudes (1.17).

The vacuum energy can be written in the form

$$
\begin{aligned}
E(g) & =-\lim _{V \rightarrow \infty} \frac{1}{V} \ln S_{V}[g, 0]=-\lim _{V \rightarrow \infty} \frac{1}{V} B_{V}[g, 0] \\
& =-g a(0)+\sum_{n=2}^{\infty} \frac{g^{n}}{n} \mathscr{E}_{n}
\end{aligned}
$$

where

$$
\begin{aligned}
a(0) & =\lim _{V \rightarrow \infty} a(V, 0)=\int_{-\infty}^{\infty} d \beta \tilde{U}(\beta) \exp \left\{-\frac{1}{2} \beta^{2} D(0)\right\} \\
\mathscr{E}_{n} & =\lim _{V \rightarrow \infty} \frac{1}{V} \sum_{j=1}^{\infty} 2 \operatorname{Re}\left[\frac{V}{a_{j}(V)}\right]^{n} \\
& =\lim _{V \rightarrow \infty} \frac{2}{V} \sum_{j=1}^{\infty} \frac{\cos n \theta_{j}(V)}{\left[\frac{r_{j}(V)}{V}\right]^{n}}
\end{aligned}
$$

As stated above, from the analysis of the perturbation series it is known that $\mathscr{E}_{n}$ is finite, i.e. the limit in (4.8) does exist, and

$$
\varlimsup_{n \rightarrow \infty}\left|\frac{\mathscr{E}_{n}}{n}\right|^{1 / n}=\frac{1}{g_{0}}
$$

where $g_{0}$ is the convergence radius of the series (4.6).

Let us consider now an amplitude of $m$ interacting particles. The connected part of this amplitude is determined by the formulas (1.16). Substituting the representation (4.4) in (1.16) and taking into account the symmetry of the function 
$B_{m}\left(V ; y_{1}, \ldots, y_{m}\right)$ one can obtain

$$
\begin{aligned}
B_{m}^{(n)}\left(V ; y_{1}, \ldots, y_{m}\right) & \\
= & \left.2 \operatorname{Re} \frac{\delta^{m}}{\delta \varphi\left(y_{1}\right) \cdot \ldots \cdot \delta \varphi\left(y_{m}\right)} \sum_{j=1}^{\infty}\left(\frac{V}{a_{j}(V, \varphi)}\right)^{n}\right|_{\varphi=0} \\
= & 2 \operatorname{Re} \sum_{i_{1} ! i_{2} ! \ldots i_{m} !} \frac{(-)^{s}(n+s-1) !}{(n-1) !} \cdot P(1, \ldots, m) \\
& \cdot \frac{1}{V^{s}} \sum_{j=1}^{\infty}\left(\frac{V}{a_{j}(V)}\right)^{n+s} \cdot\left(\frac{a_{j}^{(1)}}{1 !}\right)^{i_{1}} \cdot\left(\frac{a_{j}^{(2)}}{2 !}\right)^{i_{2}} \cdot \ldots \cdot\left(\frac{a_{j}^{(m)}}{m !}\right)^{i_{m}} .
\end{aligned}
$$

Here the summation is carried out over all positive solutions of the equation $i_{1}+2 i_{2}+3 i_{3}+\ldots+m i_{m}=m$;

$$
\begin{array}{r}
s=i_{1}+i_{2}+\ldots+i_{m} ; \\
a_{j}^{(i)}=a_{j}^{(i)}\left(V ; y_{1}, \ldots, y_{i}\right)
\end{array}
$$

$P(1, \ldots, m)$ is an operator which symmetrizes a function over variables $\left(y_{1}, \ldots, y_{m}\right)$.

Let us extract several first coefficient functions

$$
\begin{aligned}
B_{1}^{(n)}\left(V ; x_{1}\right)= & -n 2 \operatorname{Re} \frac{1}{V} \sum_{j=1}^{\infty}\left(\frac{V}{a_{j}(V)}\right)^{n+1} a_{j}^{(1)}\left(V ; x_{1}\right) \\
B_{2}^{(n)}\left(V ; x_{1}, x_{2}\right)= & -n 2 \operatorname{Re} \frac{1}{V} \sum_{j=1}^{\infty}\left(\frac{V}{a_{j}(V)}\right)^{n+1} a_{j}^{(2)}\left(V ; x_{1}, x_{2}\right) \\
& +\frac{n(n+1)}{V} 2 \operatorname{Re} \frac{1}{V} \sum_{j=1}^{\infty} \cdot\left(\frac{V}{a_{j}(V)}\right)^{n+2} a_{j}^{(1)}\left(V ; x_{1}\right) a_{j}^{(2)}\left(V ; x_{2}\right) ; \\
B_{3}^{(n)}\left(V ; x_{1}, x_{2}, x_{3}\right)= & -n 2 \operatorname{Re} \frac{1}{V} \sum_{j=1}^{\infty}\left(\frac{V}{a_{j}(V)}\right)^{n+1} a_{j}^{(3)}\left(V ; x_{1}, x_{2}, x_{3}\right) \\
+ & \frac{n(n+1)}{V} 2 \operatorname{Re} \frac{1}{V} \sum_{j=1}^{\infty} \cdot\left(\frac{V}{a_{j}(V)}\right)^{n+2} P(1,2,3) a_{j}^{(1)}\left(V ; x_{1}\right) a_{j}^{(2)}\left(V ; x_{2}, x_{3}\right) \\
- & \frac{n(n+1)(n+2)}{V^{2}} 2 \operatorname{Re} \frac{1}{V} \sum_{j=1}^{\infty} \cdot\left(\frac{V}{a_{j}(V)}\right)^{n+3} a_{j}^{(1)}\left(V, x_{1}\right) a_{j}^{(1)}\left(V, x_{2}\right) a_{j}^{(1)}\left(V, x_{3}\right)
\end{aligned}
$$

and so on. 
According to the results of perturbation theory there exist the finite limits for any $n$ and $m$ :

$$
B_{m}^{(n)}\left(x_{1}, \ldots, x_{m}\right)=\lim _{V \rightarrow \infty} B_{m}^{(n)}\left(V ; x_{1}, \ldots, x_{m}\right) .
$$

The functions $B_{m}^{(n)}\left(x_{1}, \ldots, x_{m}\right)$ are translation-invariant functions and

$$
\varlimsup_{n \rightarrow \infty}\left|B_{m}^{(n)}\left(x_{1}, \ldots, x_{m}\right)\right|^{1 / n} \leqq \frac{1}{g_{0}}
$$

since the perturbation series converges for $|g|<g_{0}$.

The following problem is to investigate the properties of the limiting functions in (4.8) and (4.12) when $V \rightarrow \infty$.

\section{Jensen Formula and the Limit $V \rightarrow \infty$}

The entire function $F_{V}(\zeta, \varphi)$ in (4.1) has zeroes at the points $a_{j}(V, \varphi)=r_{j}(V, \varphi) e^{i \theta_{J}(V, \varphi)}(j=1,2, \ldots)$. Let $n_{V, \varphi}(R)$ be the number of zeroes of this function in the circle $|\zeta| \leqq R$. We apply the Jensen formula (see, for example, [5]):

$$
\sum_{j=1}^{N(R)} \ln \frac{R}{r_{j}(V, \varphi)}=\int_{0}^{R} \frac{d r n_{V, \varphi}(r)}{r}=\frac{1}{2 \pi} \int_{0}^{2 \pi} d \theta \ln \left|F_{V}\left(R e^{i \theta}, \varphi\right)\right|
$$

where

$$
r_{N(R)}(V, \varphi)<R<r_{N(R)+1}(V, \varphi) .
$$

With the estimation (3.2) it is easy to obtain

$$
\int_{0}^{R} \frac{d r}{r} n_{V, \varphi}(r) \leqq \frac{2}{\pi} R
$$

and this estimation does not depend on $V$.

It follows from (5.2) and (5.3) that

$$
N(R) \leqq n_{V, \varphi}(R) \leqq \frac{2}{\pi} R
$$

Let us put $R=A V$ in (5.1) where $A$ is a positive number. Making use of (5.1) and (5.4) we get

$$
\frac{1}{V} \sum_{j=1}^{N(A V)} \ln \frac{A}{\left[\frac{r_{j}(V, \varphi)}{V}\right]} \leqq \frac{2}{\pi} A
$$

for any $A$. It means that there exists the limit

$$
\lim _{V \rightarrow \infty} \frac{1}{V} \sum_{j=1}^{N(A V)} \ln \frac{A}{\left[\frac{r_{j}(V, \varphi)}{V}\right]}=C(A, \varphi) \leqq \frac{2}{\pi} A .
$$

We would remind that $\varphi=$ const. 
The sum in (5.5) can be written in the form of an integral

$$
\frac{1}{V} \sum_{j=1}^{N(A V)} \ln \frac{A}{\left[\frac{r_{j}(V, \varphi)}{V}\right]}=\int_{0}^{A_{1}(V)} d u \ln \frac{A}{r_{V}(u, \varphi)}=C_{V}(A, \varphi) .
$$

Here we define the summable function $r_{V}(u, \varphi)$ such that

$$
r_{V}(u, \varphi)=\frac{r_{j}(V, \varphi)}{V} \text { for } \quad \frac{j-1}{V}<u \leqq \frac{j}{V} \quad(j=1,2, \ldots)
$$

and

$$
A_{1}(V)=\frac{N(V A)}{V} \leqq A .
$$

Now, first, the integrand in (5.7) is positive and bounded from above due to (5.7) for any $V$. Second, the limit for $V \rightarrow \infty$ exists for any $A>0$. Therefore, by the theorem about majorant convergence in the functional analysis (see, for example, [6]) there exists the limit

$$
\lim _{V \rightarrow \infty} \ln \frac{A}{r_{V}(u, \varphi)}=\ln \frac{A}{r(u, \varphi)} \in L
$$

where

$$
\lim _{V \rightarrow \infty} \frac{r_{V u}(V, \varphi)}{V}=\lim _{V \rightarrow \infty} r_{V}(u, \varphi)=r(u, \varphi) .
$$

Then the formulas (5.6) can be written in the following form

$$
\int_{0}^{A_{1}} d n \ln \frac{A}{r(u, \varphi)}=B(A, \varphi)
$$

where

$$
r\left(A_{1}, \varphi\right)=A, A_{1}=\lim _{V \rightarrow \infty} \frac{N(A V)}{V} \leqq A .
$$

It follows from the correlations (5.6), (5.10), and (5.11) that the function $r(u, \varphi)$ is positive monotonically increasing function such that for $u \rightarrow \infty$

$$
r(u, \varphi) \rightarrow \text { const } u \text {. }
$$

Besides the function $r(u, \varphi)$ is analytic in a vicinity of the point $\varphi=0$.

It is known that there exist limits

$$
\mathscr{E}_{n}=\lim _{V \rightarrow \infty} \frac{1}{V} \sum_{j=1}^{\infty} \frac{\cos n \theta_{j}(V)}{\left[\frac{r_{j}(V)}{V}\right]^{n}}
$$

and

$$
\varlimsup_{n \rightarrow \infty}\left|\frac{\mathscr{E}_{n}}{n}\right|^{1 / n}=\frac{1}{g_{0}} .
$$


It follows from these conditions that the following limit

$$
\lim _{V \rightarrow \infty} \frac{1}{V} \sum_{j=1}^{\infty}\left[\frac{V}{r_{j}(V)}\right]^{n} \quad\left(1-\cos n \theta_{j}(V)\right)
$$

must exist.

Then we obtain, by the Fischer-Riesz theorem (see, [6]), that

$$
\lim _{V \rightarrow \infty} \theta_{V u}(V)=\theta(u)
$$

where $\theta(u)$ is a summable function. Thus we have

$$
\lim _{V \rightarrow \infty} \frac{a_{V u}(V)}{V}=r(u) e^{i \theta(u)}=a(u)
$$

where $r(u)$ and $a(u)$ are summable functions.

Moreover the function $r(u)$ is bounded from below $r(u) \geqq g_{0}$ as it follows from (5.14).

Finally, the function $r(u)$ is a positive bounded from below, monotonically increasing function which increases linearly when $u \rightarrow \infty$ :

$$
\begin{aligned}
& g_{0} \leqq r(u), \\
& r(u) \underset{u \rightarrow \infty}{\rightarrow} \text { const } u .
\end{aligned}
$$

Thus the correction to the vacuum energy in an $n$-th perturbation order can be written

$$
\mathscr{E}_{n}=2 \operatorname{Re} \int_{0}^{\infty} \frac{d u}{[a(u)]^{n}}=2 \int_{0}^{\infty} \frac{d u \cos n \theta(u)}{[r(u)]^{n}} .
$$

Now let us consider the limits when $V \rightarrow \infty$ in the expressions for the coefficient functions $B_{m}^{(n)}\left(V ; x_{1}, \ldots, x_{m}\right)$ in (4.10) and (4.11). Because of analyticity of all expressions under consideration in a vicinity of the point $\varphi=0$ and existence of the limits in (4.12) and (5.11) when $V \rightarrow \infty$, we can conclude that

$$
\lim _{V \rightarrow \infty} a_{V u}^{(m)}\left(V ; x_{1}, \ldots, x_{m}\right)=a^{(m)}\left(u ; x_{1}, \ldots, x_{m}\right)
$$

where $a^{(m)}\left(u ; x_{1}, \ldots, x_{m}\right)$ is a summable function which increases linearly when $u \rightarrow \infty$. Then we obtain

$$
\begin{aligned}
B_{m}^{(n)}\left(x_{1}, \ldots, x_{m}\right) & =\lim _{V \rightarrow \infty} B_{m}^{(n)}\left(V ; x_{1}, \ldots, x_{m}\right) \\
& =-n 2 \operatorname{Re} \int_{0}^{\infty} \frac{d u a^{(m)}\left(u ; x_{1}, \ldots, x_{m}\right)}{[a(u)]^{n+1}} .
\end{aligned}
$$

Finally let us write down the formulas describing the vacuum energy and the coefficient functions

$$
\begin{aligned}
E(g) & =-g a(0)+\sum_{n=2}^{\infty} \frac{g^{n}}{n} 2 \int_{0}^{\infty} \frac{d u \cos n \theta(u)}{[r(u)]^{n}} \\
& =-g a(0)-\int_{0}^{\infty} d u \cdot\left\{\ln \left(1-\frac{2 g}{r(u)} \cos \theta(u)+\frac{g^{2}}{r^{2}(u)}\right)+\frac{2 g}{r(u)} \cos \theta(u)\right\}
\end{aligned}
$$


where

$$
\begin{aligned}
& a(0)=\int_{-\infty}^{\infty} d \beta \tilde{U}(\beta) e^{-\frac{1}{2} \beta^{2} D(0)} ; \\
& B_{m}\left(x_{1}, \ldots, x_{m}\right) \\
& \quad=g a^{(m)}\left(x_{1}, \ldots, x_{m}\right)+2 \operatorname{Re} \sum_{n=2}^{\infty} g^{n} \int_{0}^{\infty} \frac{d u a^{(m)}\left(u ; x_{1}, \ldots, x_{m}\right)}{[a(u)]^{n+1}} \\
& =g a^{(m)}\left(x_{1}, \ldots, x_{m}\right)+2 \operatorname{Re} g^{2} \int_{0}^{\infty} \frac{d u a^{(m)}\left(u ; x_{1}, \ldots, x_{m}\right)}{[a(u)]^{2}[a(u)-g]}
\end{aligned}
$$

where

$$
a^{(m)}\left(x_{1}, \ldots, x_{m}\right)=a_{m} \int d y \prod_{j=1}^{m} \delta\left(y-x_{j}\right), a_{m}=\int_{-\infty}^{\infty} d \beta \tilde{U}(\beta)(i \beta)^{m} e^{-\frac{1}{2} \beta^{2} D(0)} .
$$

These representations are valid for $|g|<g_{0}$. Owing to (5.12) and (5.20) the integral in (5.23) is well defined.

The next problem consists in the continuation of all functions (5.22) and (5.23) into the region $|g|>g_{0}$. For this aim we have to know the phase $\theta(u)$ of the zeroes $a(u)=r(u) e^{i \theta(u)}$.

\section{Carleman Formula and the Location of Zeroes}

If a function $F(z)$ is analytic and of finite degree in the right half-plane, it has zeroes at the points $a_{k}=r_{k} e^{i \theta_{k}}$ and $F(0)=1$, then the following Carleman formula

$$
\begin{aligned}
& \sum_{\operatorname{Re} a_{k}>0} \frac{\cos \theta_{k}}{r_{k}}=\frac{1}{2 \pi} \int_{-\infty}^{\infty} \frac{d y}{y^{2}} \ln |F(i y) F(-i y)| \\
& +\lim _{R \rightarrow \infty} \frac{1}{\pi R} \int_{-\frac{\pi}{2}}^{\frac{\pi}{2}} d \theta \cos \theta \ln \left|F\left(\operatorname{Re}^{i \theta}\right)\right|-\frac{1}{2} \operatorname{Re} F^{\prime}(0)
\end{aligned}
$$

is valid for it (see, for example, [5]).

Let us define the function

$$
F_{V}(\zeta, b)=\frac{F_{V}(\zeta+b)}{F_{V}(b)} \text {. }
$$

This function $F_{V}(\zeta, b)$ has zeroes at the points

$$
\zeta=a_{j}(V)-b=r_{j}(V) e^{i \theta_{j}(V)}-b .
$$

Applying the Carleman formula to this function $F_{V}(\zeta, b)$ we obtain

$$
\sum_{\operatorname{Re}\left(a_{j}(V)-b\right)>0} \operatorname{Re} \frac{1}{a_{j}(V)-b}=J_{1}(V)+J_{2}(V)+J_{3}(V)
$$

where

$$
\begin{aligned}
& J_{1}(V)=\frac{1}{2 \pi} \int_{-\infty}^{\infty} \frac{d y}{y^{2}} \ln \left|F_{V}(i y, b) F_{V}(-i y, b)\right|, \\
& J_{2}(V)=\lim _{R \rightarrow \infty} \frac{1}{\pi} \int_{-\frac{\pi}{2}}^{\pi^{2}} d \theta \cos \theta \ln \left|F_{V}\left(R e^{i \theta}, b\right)\right|, \\
& J_{3}(V)=-\frac{1}{2} F_{V}^{\prime}(0, b) .
\end{aligned}
$$


We choose $b=g_{1} V$ where $g_{1}>0$. Then we obtain for the left part of (6.4) in the limit $V \rightarrow \infty$ :

$$
\begin{aligned}
& \lim _{V \rightarrow \infty} \sum_{\operatorname{Re}\left(a_{j}(V)-V g_{1}\right)>0} \operatorname{Re} \frac{1}{a_{j}(V)-g_{1} V} \\
& =\lim _{V \rightarrow \infty} \frac{1}{V} \sum_{\operatorname{Re}\left(\frac{a_{j}(V)}{V}-g_{1}\right)>0} \operatorname{Re} \frac{1}{\left(\frac{a_{j}(V)}{V}\right)-g_{1}} \\
& =\int_{G\left(g_{1}\right)} d u \operatorname{Re} \frac{1}{r(u) e^{i \theta(u)}-g_{1}} \geqq 0
\end{aligned}
$$

where the integration is performed over the region

$$
G\left(g_{1}\right)=\left\{u: \operatorname{Re}\left[r(u) e^{i \theta(u)}-g_{1}\right]>0\right\} .
$$

For the right member of (6.4), making use of the estimations of Section 3 we can obtain :

$$
\begin{aligned}
& J_{1}=\lim _{V \rightarrow \infty} J_{1}(V)=0, \\
& J_{2}=\lim _{V \rightarrow \infty} J_{2}(V)=\frac{1}{2}, \\
& J_{3}=\lim _{V \rightarrow \infty} J_{3}(V)=-\frac{1}{2} .
\end{aligned}
$$

It means that for any $g_{1}>0$

$$
\int_{G\left(g_{1}\right)} \frac{d u\left[r(u) \cos \theta(u)-g_{1}\right]}{r^{2}(u)-2 g_{1} r(u) \cos \theta(u)+g_{1}^{2}}=0 .
$$

The integrand in (6.11) is positive, then the integral (6.11) can be equal to zero only in the case if

$$
\frac{\pi}{2} \leqq \theta(u) \leqq \pi
$$

For the odd interaction functions $U(\varphi)=-U(-\varphi)$ we obtain

$$
\theta(u)=\frac{\pi}{2}
$$

because the function $F_{V}(g, 0)$ is even: $F_{V}(g, 0)=F_{V}(-g, 0)$.

For the even interaction functions $U(\varphi)=U(-\varphi)$ we can conclude only that

$$
\frac{\pi}{2} \leqq \theta(\mathrm{u}) \leqq \pi .
$$

Below we will consider all formulas for the odd interaction functions $U(\varphi)$ only. The vacuum energy (5.22) and the coefficient functions (5.23) can be written in this case as :

$$
\begin{aligned}
& E(g)=-\int_{0}^{\infty} d u \ln \left(1+\frac{g^{2}}{r^{2}(u)}\right) \\
& B_{m}\left(x_{1}, \ldots, x_{m}\right)=g a^{(m)}\left(x_{1}, \ldots, x_{m}\right)+2 g^{2} \operatorname{Re} i \int_{0}^{\infty} \frac{d u a^{(m)}\left(u ; x_{1}, \ldots, x_{m}\right)}{r^{2}(u)[r(u)-i g]}
\end{aligned}
$$


or

$$
T_{m}\left(p_{1}, \ldots, p_{m}\right)=g \tilde{a}_{m}-2 g^{2} \operatorname{Im} \int_{0}^{\infty} \frac{d u[r(u)+i g] \tilde{a}^{(m)}\left(u ; p_{1}, \ldots, p_{m}\right)}{r^{2}(u)\left[r^{2}(u)+g^{2}\right]}
$$

where the connection between $\tilde{a}_{m}$ and $a^{(m)}$ is given by the formula (1.16).

From these formulas we can see that singularities in the complex $g$-plane are on the imaginary axis. There are no singularities on the real axis. These formulas (6.15) and (6.17) give the representations of our functions for any positive constant $g$.

It follows from the conditions (5.12) and (5.20) that for $g \rightarrow \infty$

$$
\begin{aligned}
& E(g)=O(g), \\
& T_{m}\left(p_{1}, \ldots, p_{m}\right)=O(g),
\end{aligned}
$$

i.e. all matrix elements of the $S$-matrix increase linearly when $g \rightarrow \infty$.

\section{Green Function in the Physical Region}

The representation (6.18) for the amplitudes $T_{m}$ was obtained in the Euclidean region of momentum variables $\left(p_{1 E}, \ldots, p_{m E}\right)$, where these amplitudes well decrease for $p_{j E}^{2} \rightarrow+\infty$. It should be noted that above we did not introduce any special notations for the Euclidean momenta because we considered all expressions in the Euclidean space only. After continuation in the physical region $p_{j E}^{2}=-p^{2}$ any amplitudes $T_{m}\left(p_{1}, \ldots, p_{m}\right)$ where $p_{j}$ are physical momenta increase for $p_{j}^{2} \rightarrow+\infty$ in each perturbation order as $\exp \left\{(n-1)\left(p^{2} l^{2}\right)^{\varrho}\right\}$ where $n$ is the perturbation order and $\varrho$ is the order of the nonlocal form factor $K\left(p^{2}\right)$. The problem is to find the behaviour of the amplitudes (6.17) in the physical region for $p_{j}^{2} \rightarrow+\infty$. In this section we will prove that the twopoint Green function is bounded for $p^{2} \rightarrow+\infty$. The analogous arguments lead to the conclusion that any amplitudes $T_{m}\left(p_{1}, \ldots, p_{m}\right)$ are bounded in the physical region.

Let us consider the twopoint Green function $\left(p^{2}=-p_{E}^{2}\right)$

$$
G\left(g, p^{2}\right)=T_{2}\left(p_{E},-p_{E}\right)=\int d x_{E} e^{i p_{E} x_{E}} B_{2}\left(\frac{x_{E}}{2},-\frac{x_{E}}{2}\right)
$$

for the odd interaction functions $U(\varphi)$. Making use of the representation (6.17) we obtain

$$
G\left(g, p^{2}\right)=-2 g^{2} \operatorname{Im} \int_{0}^{\infty} \frac{d u[r(u)+i g] \tilde{a}^{(2)}\left(u ; p_{E},-p_{E}\right)}{r^{2}(u)\left[r^{2}(u)+g^{2}\right]} .
$$

On the other hand the Green function can be represented according to (1.17) as a perturbation expansion:

$$
G\left(g, p^{2}\right)=\sum_{n=1}^{\infty} g^{2 n} G_{2 n}\left(p^{2}\right)
$$

where

$$
\begin{aligned}
G_{2 n}\left(p^{2}\right)=\frac{1}{(2 n) !} \int_{-\infty}^{\infty} d \beta_{1} \tilde{U}\left(\beta_{1}\right) e^{-\frac{1}{2} \beta_{1}^{2} D(0)} \\
\cdot \ldots \cdot \int_{-\infty}^{\infty} d \beta_{2 n} \tilde{U}\left(\beta_{2 n}\right) e^{-\frac{1}{2} \beta_{2 n}^{2} D(0)} \\
\cdot(2 n)^{4} \int d x_{1 E} \ldots \int d x_{2 n E} \delta\left(x_{1 E}+\ldots+x_{2 n E}\right) \\
\cdot\left\{\prod_{i<j}\left(1+w\left(\beta_{i} \beta_{j}, x_{i E}-x_{j E}\right)\right)\right\}_{c} \sum_{i, j=1}^{2 n} \beta_{i} \beta_{j} e^{i p_{E}\left(x_{i E}-x_{J E}\right)},
\end{aligned}
$$


So long as the perturbation series (7.3) contains only the even degrees of $g$, the representation (7.2) should be written in the form

$$
G\left(g, p^{2}\right)=2 g^{2} \int_{0}^{\infty} \frac{d u \operatorname{Im} \tilde{a}^{(2)}\left(u ; p_{E},-p_{E}\right)}{r(u)\left[r^{2}(u)+g^{2}\right]} .
$$

Let us introduce the new variables

$$
t=\frac{g_{0}}{r(u)}, \lambda^{2}=\frac{g^{2}}{g_{0}^{2}}, p^{2}=-p_{E}^{2}=s .
$$

Then the representation (7.5) can be rewritten in the form

$$
G\left(g, p^{2}\right)=\lambda^{2} \int_{-1}^{1} \frac{d t A(t, s)}{1+\lambda^{2} t^{2}}
$$

where $A(t, s)$ is a bounded summable function of $t$ in the interval $-1 \leqq t \leqq 1$.

Expanding the integrand in (7.7) in the perturbation series and comparing with (7.4) we obtain

$$
G_{2 n}(s)=\frac{(-)^{n}}{g_{0}^{2 n}} \int_{-1}^{1} d t t^{2(n-1)} A(t, s) .
$$

It follows from the conditions (1.18) and (4.13) that

$$
\varlimsup_{n \rightarrow \infty}\left|G_{2 n}(s)\right|^{1 / 2 n} \leqq \frac{1}{g_{0}}
$$

for all Euclidean momenta $p_{E}^{2}$, i.e. for $p^{2}=s<0$.

Our problem is to continue the Green function $G(g, s)$ into the physical region of $p^{2}$, i.e. into the region $p^{2}=s>0$.

The analysis of the perturbation series performed in [1] showed that for $s \rightarrow+\infty$

$$
G_{2 n}(s)=O\left(\exp \left\{(2 n-1)\left(s l^{2}\right)^{\varrho}\right\}\right)
$$

where $\varrho$ is the order of the nonlocal form factor $K\left(p^{2} l^{2}\right)$.

The function $A(t, s)$ in (7.7) can be expanded in the series over the orthogonal Chebyshev polynomials of the second kind (see, for example, [7])

$$
A(t, s)=\sqrt{1-t^{2}} \sum_{m=1}^{\infty} A_{2 m}(s) U_{2 m}(t)
$$

where the polynomials $U_{2 m}(t)$ are defined by the following generating function

$$
\frac{1}{1-2 t w+w^{2}}=\sum_{m=0}^{\infty} w^{m} U_{m}(t)
$$

and satisfy the orthogonality conditions

$$
\int_{-1}^{1} d t \sqrt{1-t^{2}} U_{m}(t) U_{m^{\prime}}(t)=\frac{\pi}{2} \delta_{m m^{\prime}} .
$$


Comparing the formulas (7.8), (7.10), and (7.11) we obtain

$$
A_{2 m}(s)=O\left(\exp \left\{(2 m+1)\left(s l^{2}\right)^{\circ}\right\}\right)
$$

when $s \rightarrow \infty$.

We make use of the standard procedure for the analytical continuation into the region of positive real $s$. First, it should be noted that the series (7.11) defines the analytical function with respect to $s$ in a vicinity of the semi-axis $s \leqq 0$. Let us introduce the function

$$
h(t, s)=\frac{2}{\pi} \sum_{m=1}^{\infty} e^{-(2 m+1) \varkappa} A_{2 m}(s) U_{2 m}(t)
$$

where $x=\left[\left(s_{0}+s\right) l^{2}\right]^{\circ}$ and $s_{0}>\varepsilon>0$.

This series defines the bounded analytical function for $-1 \leqq t \leqq 1$ and $-s_{0} \leqq \operatorname{Re} s \leqq 0$. Then we can obtain from (7.15) that

$$
A_{2 m}(s)=e^{(2 m+1) x} \int_{-1}^{1} d \tau \sqrt{1-\tau^{2}} h(\tau, s) U_{2 m}(\tau)
$$

Substituting (7.16) in (7.11) and (7.7) we obtain

$$
\begin{aligned}
G(g, s)= & \lambda^{2} e^{\varkappa} \int_{-1}^{1} \frac{d t \sqrt{1-t^{2}}}{1+\lambda^{2} t^{2}} \sum_{m=1}^{\infty} U_{2 m}(t) e^{2 m \varkappa} \\
& \cdot \int_{-1}^{1} d \tau \sqrt{1-\tau^{2}} h(\tau, s) U_{2 m}(\tau) .
\end{aligned}
$$

The order of integration over $t$ and summation over $m$ can be changed. Making use of the equality

$$
\int_{-1}^{1} \frac{d t \sqrt{1-t^{2}} U_{2 m}(t)}{1+\lambda^{2} t^{2}}=2 \pi(-)^{m}\left(\sqrt{1+\lambda^{2}}-1\right)\left(\frac{\sqrt{1+\lambda^{2}}-1}{\sqrt{1+\lambda^{2}}+1}\right)^{m}
$$

it can be observed that in the region

$$
\left|\frac{\sqrt{1+\lambda^{2}}-1}{\sqrt{1+\lambda^{2}}+1} e^{2 x}\right|<1
$$

the series over $m$ in (7.17) converges absolutely and we can change the order of summation over $m$ and integration over $\tau$. After some transformations we obtain

$$
\begin{aligned}
G(g, s)= & \pi \lambda^{2}\left(\sqrt{1+\lambda^{2}}-1\right)^{2} \int_{-1}^{1} \frac{d \tau \sqrt{1-\tau^{2}} h(\tau, s)}{\lambda^{2} \tau^{2}+\left(\operatorname{ch} x-\sqrt{1+\lambda^{2}} \operatorname{sh} x\right)^{2}} \\
& \cdot e^{x}\left[e^{x}\left(\operatorname{ch} x-\sqrt{1+\lambda^{2}} \operatorname{sh} x\right)-2 \tau^{2}\left(\sqrt{1+\lambda^{2}}+1\right)\right] .
\end{aligned}
$$

This representation (7.20) is obtained in the region (7.19) but it is valid in the region of any positive $s>-s_{0}$. Really, the structure of the integrand is that the Green function $G(g, s)$ has no singularities for

$$
\operatorname{ch} x-\sqrt{1+\lambda^{2}} \operatorname{sh} x=0 .
$$


The nearest singularity of $G(g, s)$ arises when

$$
\lambda^{2}+\left(\operatorname{ch} x-\sqrt{1+\lambda^{2}} \operatorname{sh} x\right)^{2}=0
$$

from which

$$
\varkappa=\left[\left(s+s_{0}\right) l^{2}\right]^{\varrho}= \pm i \frac{\pi}{2}+\frac{1}{2} \ln \frac{\sqrt{1+\lambda^{2}}+1}{\sqrt{1+\lambda^{2}}-1} .
$$

Thus the analytical properties of the Green function in a vicinity of the real axis of are defined by the properties of function $h(\tau, s)(7.15)$, i.e. by the properties of the perturbation series. The summation of the perturbation series does not lead to any new additional singularities in the physical region of momentum variables.

Now let us consider the behaviour of the Green function for high energies in the physical region. Making use of the representation (7.20) for $s \rightarrow+\infty$ we obtain

$$
G(g, s) \simeq 8 \pi \frac{\lambda^{2}-1}{\lambda^{2}}\left(\sqrt{1+\lambda^{2}}+1\right)^{2} e^{-x} \int_{-1}^{1} d \tau \sqrt{1-\tau^{2}} h(\tau, s) \tau^{2}=O\left(e^{-\varkappa}\right)
$$

because the function $h(\tau, s)$ is bounded for $s \rightarrow \infty$. Thus, the Green function is bounded for high energies though it increases very rapidly in each perturbation order.

The analogous calculations can be performed for any $S$-matrix elements.

Acknowledgements. I would like to thank Prof. D. I. Blokhintsev, B. N. Valuev, V. A. Zagrebnov, V. V. Priezjev, and V. K. Fedjanin for discussions.

\section{References}

1. Alebastrov,V.A., Efimov,G.V.: Commun. math. Phys. 31, 1 (1973); 38, 11 (1974)

Efimov,G.V.: Nonlocal interactions of quantized fields. Moscow: Nauka 1977

2. Petrina,D.Ja., Skripnik, V.I. : Sovjet Teor. Math. Phys. 8, 368 (1971)

3. Basuev, A. G.: Sovjet Teor. Math. Phys. 16, 281 (1973)

4. Hardy,G.H., Littlewood,J.E., Polya,G.: Inequalities. London: Cambridge University Press 1951

5. Titchmarsh,E.C.: The theory of functions. London: Oxford University Press 1939

6. Shilov, G.E., Gurevitch, B.L. : Integral, measure and derivative. Moscow: Nauka 1967

7. Szego,G.: Orthogonal polynomials. New York: American Mathematical Society 1959

Communicated by R. Haag

Received June 26, 1977 